\title{
COAGULACION DE COMPLEJOS DE HIERRO (III) EN LAS AGUAS DEL RIO TARUCACHI, TACNA
}

\author{
Leoncio Molina Vásquez ${ }^{1}$
}

\section{INTRODUCCION}

Las aguas del río Tarucachi (situado en la provincia de Tarata, departamento de Tacna) deorigen geotermal tienen una concentración de hierro entre 3,25 y 4,50 mg $\mathrm{dm}^{-3}$; y de arsénico entre $0,125 \mathrm{mg} \mathrm{dm}^{-3}$ a un $\mathrm{pH}$ que fluctúa entre 2,35 y 3,45 y que mediante reacciones experimentales con $\mathrm{CaCO}_{3}$ (caliza) es modificado, logrando formar dispersiones coloidales de hierro de estabilidad extensa, cuyas partículas varían en tamaño con la elevación del $\mathrm{pH}$. A medida que el $\mathrm{pH}$ es neutro 0 ligeramente ácido se observa que la coagulaciónes más rápida e involucra iones metálico y arsénico, por su gran capacidad de absorción.

Las principales características y análisis físico químicos del agua del río Tarucachi se aprecian en las tablas No. 01 y No. 02.

TABLA No. 1: Características del agua del río Tarucachi.

\begin{tabular}{|l|c|}
\hline PARAMETRO & \\
\hline Aspecto & Con gránulos rojizos \\
Turbidez & $23-3,0$ UNT \\
$\mathrm{pH}$ & $2,35-3,0$ \\
Conductividad & $1,180 \mu / \mathrm{CM}$ \\
Alcalinidad & 0 \\
Fenolftaleina & 0 \\
Alcalinidad Total & 0 \\
$\mathrm{CO}_{2}$ libre & $182 \mathrm{pp} . \mathrm{m}$ \\
\hline
\end{tabular}

Fuente : Análisis LFQ - UNJBG.

1. Magíster e ingeniero químico
TABLA No. 2: Análisis físico-químico del agua del río Tarucachi.

\begin{tabular}{|l|r|}
\hline \multicolumn{1}{|c|}{ SUSTANCIA } & $\begin{array}{r}\text { CONCENTRACION } \\
\mathrm{mg} / \mathrm{dm}^{3}\end{array}$ \\
\hline $\mathrm{Na}$ (K incluido) & $86,73-100,15$ \\
$\mathrm{Ca}$ & $168-416$ \\
$\mathrm{Mg}$ & $28,8-32,6$ \\
$\mathrm{Fe}$ Total & $3,25-4,5$ \\
$\mathrm{Fe}$ Soluble & $0,55-0,80$ \\
$\mathrm{Cu}$ & $0,45-0,70$ \\
$\mathrm{Cl}_{2}$ & $20,00-21,00$ \\
$\mathrm{SO}_{4}$ & $539,04-$ \\
$\mathrm{As}^{2}$ & $0,059-0,125$ \\
\hline
\end{tabular}

Fuente : Análisis LFQ - UNJBG.

\section{REACCIONESDE HIERRO}

\subsection{COMPORTAMIENTODEL HIERRO ENELAGUA DELRIOTARUCACHI}

Las soluciones de Fe (III) son amarillas o de color pardo rojizo (la coloración diferente de las soluciones de las sales de hierro depende del tipo de complejos que existen en las soluciones).

El hidróxido de $\mathrm{Fe}$ (III), prácticamente no posee propiedades anfóteras, por eso es insoluble en un exceso alcali. La precipitación de $\mathrm{Fe}(\mathrm{OH})_{3}$ se consigue con un $\mathrm{pH}$ mayor a 3,5 como un complejo (1)

$$
\mathrm{Fe}(\mathrm{OH})_{3}\left(\mathrm{Fe}_{2} \mathrm{O}_{3}\right) \cdot \mathrm{XH}_{2} \mathrm{O}
$$

EIFe(III), enfrío, forma un complejo soluble en agua cuando se encuentra a un $\mathrm{pH}$ menor de 3,5.

Los carbonatos de metales alcalinos y alcalinotérreos forman con $\mathrm{Fe}$ (III) un precipitado 
pardo de sales básicas, que si se calentaran se convertirían en hidróxido.

\subsection{REACCIONES DE LOIONES Fe (II)}

Las sales de Fe (II) tienen un color verde pálido, pero en soluciones diluidas son incoloras.

En condiciones ordinarias, pueden tener una oxidación parcial y el precipitado mostrar un color turbio. En cambio, cuando se expone al aire, se le puede observar cómodamente filtrándolo. El producto final de la oxidación es $\mathrm{Fe}(\mathrm{OH})_{3}$. LoS precipitados de $\mathrm{Fe}(\mathrm{OH})_{2}$ y $\mathrm{Fe}(\mathrm{OH})_{3}$ se disuelven en ácidos, pero son insolubles en alcalis.

Los carbonatos metales alcalinos dan con $\mathrm{Fe}$ (II) un precipitado blanco de $\mathrm{FeCO}_{3}$, el cual, muy pronto, expuesto al aire, toma un color pardo, debido a su oxidación, produciéndose $\mathrm{Fe}(\mathrm{OH})_{3}$.

$$
4 \mathrm{Fe}(\mathrm{OH})_{3} \downarrow+6 \mathrm{H}_{2} \mathrm{O}+3 \mathrm{O}_{2} \rightarrow 4 \mathrm{CO}_{2} \uparrow+4 \mathrm{Fe}(\mathrm{OH})_{3} \downarrow
$$

$\mathrm{El}$ ión $\mathrm{Fe}^{2+}$ es un reductor muy fuerte y capaz de oxidarse bajo la acción de una serie de oxidantes, tales como $\mathrm{H}_{2}, \mathrm{O}_{2}$ y otros en medio ácido, como ocurre con los sulfuros de las aguas que tienen $\mathrm{NO}_{3}$

$\mathrm{FeS}+4 \mathrm{H}^{+}+\mathrm{NO}_{3} \rightarrow \mathrm{Fe}^{3+}+\mathrm{S} \downarrow+2 \mathrm{H}_{2} \mathrm{O}+\mathrm{NO} \uparrow$

El hierro en el agua, cuando el $\mathrm{pH}$ es mayor que 3,5 , se encontrará en forma de $3^{3+}$. Por esta razón, la precipitación de $\mathrm{Fe}^{3+}$ es mayorque el $\mathrm{Fe}^{2+}$.

\subsection{HIDROLISIS DE Fe $\mathrm{e}^{3+}$}

Los iones metálicos hidratados son ácidos en el sentido dado por Brönsted.

El equilibrio ácido base para el ión férrico es:

$$
\mathrm{Fe}\left(\mathrm{H}_{2} \mathrm{O}\right) 6^{3+}+\mathrm{H}_{2} \mathrm{O} \rightarrow\left[\mathrm{Fe}\left(\mathrm{H}_{2} \mathrm{O}\right)_{5}(\mathrm{OH})\right]^{2+}+\mathrm{H}_{3} \mathrm{O}^{+}
$$

Altransferir un protón a una molécula de agua, una agua coordinada se convierte en hidróxido; la base conjugada puede entoncestransferirun protón.

$$
\left[\mathrm{Fe}\left(\mathrm{H}_{2} \mathrm{O}\right)_{5} \mathrm{OH}\right]^{2+}+\mathrm{H}_{2} \mathrm{O} \rightarrow\left[\mathrm{Fe}\left(\mathrm{H}_{2} \mathrm{O}\right)_{4}(\mathrm{OH})_{2}\right]^{2+}+\mathrm{H}_{3} \mathrm{O}^{+}
$$

Los complejos hidroférricos tienen una enorme tendencia a polimerización produciendo reacción, asi como:

$$
2\left[\mathrm{Fe}\left(\mathrm{H}_{2} \mathrm{O}\right)_{5}(\mathrm{OH})\right]^{2+} \rightarrow\left[\mathrm{Fe}\left(\mathrm{H}_{2} \mathrm{O}\right)_{8}(\mathrm{OH})_{2}\right]^{4+}+2 \mathrm{H}_{2} \mathrm{O}
$$

Los iones metálicos se encuentran ligados probablemente mediante dos puentes hidroxo.

Este dímero puede sufrir reacciones hidrolícas adicionales y formar complejos mayores de hidróxidos los cuales formarian entonces más puentes hidroxo.

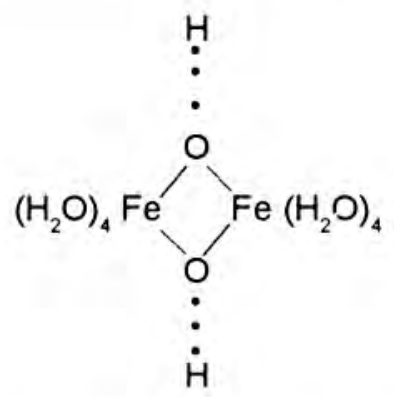

$$
\left[\mathrm{Fe}_{2}\left(\mathrm{H}_{2} \mathrm{O}\right)_{8}(\mathrm{OH})\right]^{4+}+\mathrm{H}_{2} \mathrm{O} \rightarrow\left[\mathrm{Fe}_{2}\left(\mathrm{H}_{2} \mathrm{O}_{7}(\mathrm{OH})_{3}\right]^{3+}+\mathrm{H}_{3} \mathrm{O}^{+}\right.
$$

Una secuencia de reacciones hidrolíticas acompañada probablemente de reacciones de deshidratación, conduce a una coordinación progresiva del ión férrico con iones hidróxido.

Conforme disminuye la carga de las especies, elFe (III) por la coordinación con los grupos hidroxo, hace disminuir la repulsión entre iones y se da una mayor tendencia hacia la polimerización. Asi se forman polímeros hidroxocoloidales y precipitados insolubles de óxido férrico hidratado.

La posición de una estructura polimérica por parte del óxido férrico, se basa en la observación de que el momento magnético del óxido de hierro hidratado, menor que el de las sales de Fe (III). Debido a que los complejos catiónicos hidroxo férricos prevalecen ensoluciones ligeramente ácidas o neutras se forman coloides de óxido férrico cargados positivamente en esta gama de $\mathrm{pH}$.

\subsection{COAGULACION DEL Fe $\mathrm{Fe}^{3+}(10)$}

Los complejos hidroxo polinucleares disueltos que son intermedios en la transición de los iones de hierro, son coagulantes eficientes.

a) $\mathrm{A}$ valores de $\mathrm{pH}$ menores de $3 \mathrm{el} \mathrm{Fe}^{3+}$ no se encuentra hidrolizado.

b) A valores más altos de $\mathrm{pH}$. Los complejos hidroxo polinucleares, altamente cargados se forman y se adhieren fuertemente sobre las superficies de los coloides. Por consiguiente son capaces de invertir la carga de los coloides cuando se encuentran a una concentración algo más alta, esto se incrementa con la sílice en suspensión.

c) A partir de a concentración de inversión de la cara se concluye que las especies hidrolizadas y no el $\mathrm{Fe}^{3+}$, causan la inversión de la carga.

d) A concentraciones más elevadas de Fe (III), se precipita óxido férrico. 


\subsection{POTENCIAL ZETA}

El equilibrio químico especifico de las sustancias coloidales y disueltas así como la interacción química entre las impurezas y los iones metálicos son, algunas veces, más importantes que las fuerzas electrostáticas que compactan la fuerza difusa de la capa doble. La absorción de los iones metálicos hidrolizados sobre los coloides dispersos es un ejemplo.

El aumento de la concentración de iones origina la relativa facilidad para obtener la coagulación de óxidos e hidróxidos férricos en agua son sales, alcalino y alcalino térreas, en comparación con el agua destilada.

La presencia de iones $\mathrm{SO}_{4}{ }_{4}^{2}$-proporciona ayuda en la coagulación de los coloides cargados positivamente con óxidos de hierro.

\subsection{PRECIPITACION}

La precipitación es esencialmente un proceso opuesto a la disolución; donde se puede requerir cantidades superiores a las calculadas para mantener el comportamiento deseado. Comola regla, los precipitantes se deben seleccionar entre:

a) Compuestos químicos que se encuentran normalmente en la naturaleza como carbonatos.

b) Cuerpos químicos relativamente insolubles como hierroy aluminio trivalente.

c) Materiales químicos que no producen residuos tóxicos, como por ejemplo el $\mathrm{Ba}^{2+}$.

La mayor parte de los cationes multivalentes en el agua se pueden precipitar en cantidades predecibles como carbonatos o hidróxidos. Porque su solibilidad gobierna a procesos importantes de tratamiento, incluyendo la estabilización química y la precipitación del $\mathrm{Ca}^{2+} \mathrm{y} \mathrm{Mg}^{2+}$, así como de otros cationes en la suavización de aguas.

Sin embargo, no basta conocer la magnitud del producto de solubilidad. Las especies que se disuelven cuando se agrega $\mathrm{CaCO}_{3(\mathrm{~S})}$ al agua son $\mathrm{Ca}^{2+}, \mathrm{y} \mathrm{CO}_{3}{ }^{2-} \mathrm{y} \mathrm{HCO}_{3}-$; los productos de su reacción con el agua son predominantemente, $\mathrm{HCO}_{3}-\mathrm{y} \mathrm{OH}^{-}$ . En términos generales $\mathrm{CaCO}_{3}$ reacciona con los iones hidrógeno o con un ácido en la siguiente forma:

$$
\mathrm{CaCO}_{3(\mathrm{~s})}+\mathrm{H}^{+} \rightarrow \mathrm{C}^{2+}+\mathrm{HCO}_{3}^{-}
$$

Es obvio que al disminuir el $\mathrm{pH}$ mediante la adición de un ácido aumenta la solubilidad de $\mathrm{CaCO}_{3}$, mientrasquela elevación del pH através del aporte de una base, disminuye la solubilidad. De hecho la elevación del pHgobierna la remoción del $\mathrm{Ca}^{2+}$ mediante el proceso de suavización por cal / carbonato.

\section{TRABAJOEXPERIMENTAL}

\subsection{PROGRAMA DETRABAJO}

El programa elaborado para el trabajo experimentalfue:

a) Enumeración de las principales especies que existen en la solución en el equilibrio; tablas No. 01 y 02.

b) Diseño de un canal experimental con un emboquillado de caliza ; figuras No. 1, 2 y 3.

c) Establecimiento de los diversos equilibrios de los iones principales, para formar las redes coloidales de hierro (III).

d) Reacciones que identifican a los componentes del soluto en las aguas en contacto con el $\mathrm{CaCO}_{3}$ (caliza).

e) Se calculó las concentraciones de equilibrio de los solutos del $\mathrm{CaCO}_{3}$ con variación del pH y del tiempo en agua del río Tarucachi.

f) Se construyó un canal experimental en sistema abierto a la atmósfera, con caliza emboquillada, en este caso no se desprecia el $\mathrm{CO}_{2}$ de la atmósfera, si no que se trata al $\mathrm{H}_{2} \mathrm{CO}_{3}$ en soluciones, como ácido no volátil.

\subsection{RESULTADOSEXPERIMENTALES}

a) En el laboratorio se demuestra que la estabilidad de Fe (III) y su precipitación se modifican cuando se agrega o entra en contacto la caliza con el agua de $\mathrm{pH}$ ácido del río Tarucachi; las especies que se forman son:

$$
\begin{aligned}
& \mathrm{CaCO}_{3(\mathrm{~s})}+\mathrm{H}_{2} \mathrm{O} \rightarrow \mathrm{Ca}(\mathrm{OH})_{2}+\mathrm{CO}_{2} \\
& \mathrm{CaCO}_{3(\mathrm{~s})}+\mathrm{H}^{+} \rightarrow \mathrm{Ca}_{(\mathbf{a q})}{ }^{2+}+\mathrm{HCO}_{3(\mathrm{aq})}
\end{aligned}
$$

Es obvio que al disminuirel $\mathrm{pH}$, la solubilidad del $\mathrm{CaCO}_{3(8)}$ aumenta, mientras que al disminuir el $\mathrm{pH}$ es a la inversa, tal como se aprecia en la tabla No. 3.

\section{b) Coagulación del hierro}

Los complejos hidroxo polinucleares disueltos intermedios en la transición de los iones de hierro a óxidos hidratados, son coagulantes eficientes. Bajo las condiciones favorables de $\mathrm{pH}$, temperatura y tiempo de añejamiento con la caliza, los productos de hidrólisis de los iones metálicos 
TABLA No. 3

\begin{tabular}{|c|r|c|c|}
\hline No & $\mathrm{t}(\mathrm{min})$ & $\mathrm{pH}$ & $\mathrm{Ca}^{2+} \mathrm{mg} / \mathrm{dm}^{3}$ \\
\hline 1 & 0 & 3,35 & 168 \\
2 & 0,5 & 5,90 & 212 \\
3 & 1,0 & 6,50 & 216 \\
4 & 1,5 & 6,80 & 236 \\
5 & 3,0 & 6,80 & 236 \\
6 & 5,0 & 6,85 & 236 \\
7 & 10,0 & 6,90 & 248 \\
8 & 15,0 & 7,00 & 264 \\
\hline
\end{tabular}

Fuente : Análisis LFQ - UNJBG.

poseen una mayor carga que los iones metálcos mismos se absorben fuertemente sobre las dispersiones coloidales. De acuerdo a la experimentación realizada:

- A pH menores de 3,5 el Fe (III) no se encuentra hidrolizado, pero a pH mayores de 4 todo el hierro estrivalente.

- A valores más altos de pH 4 se forman los complejos hidroxo polinucleares de Fe (III) observados al microscopio.

- Allegar a pH6,8 los complejos altamente cargados se adhieren fuertemente sobre la superficie de los coloides.

TABLA No. 4: Hierro (III) soluble en el agua del río Tarucachi

\begin{tabular}{|c|c|c|}
\hline No. & \multicolumn{1}{|c|}{$\mathrm{pH}$} & $\mathrm{Fe} \mathrm{mg} \mathrm{dm}^{-3}$ \\
\hline 1 & $<3,5$ & 4,45 \\
2 & 3,5 & 0,66 \\
3 & 5,90 & 0,44 \\
4 & 6,65 & 0,25 \\
5 & 6,85 & 0,06 \\
6 & 7,00 & 0,013 \\
\hline
\end{tabular}

\section{c) Arsénico en el agua del río Taruchachi}

El arsénico proveniente de las arsenopiritas de la caja donde discurren las aguas, al saturarse con aire se oxidan a arsénico ( $V$ ) porque el arsénico (III) tiene una breve existencia en esas condiciones.

Por la presencia del $\mathrm{H}_{2} \mathrm{~S}$ de las aguas geotermales que ingresan al río Tarucachi, tenemos:

$$
2 \mathrm{~A}_{\mathrm{S}} \mathrm{O}_{4}{ }^{3-}+5 \mathrm{H}_{2} \mathrm{~S}+6 \mathrm{H}^{+} \rightarrow \mathrm{As}_{2} \mathrm{~S}_{3}+2 \mathrm{~S}+8 \mathrm{H}_{2} \mathrm{O}
$$

La reducción del $\mathrm{A}_{\mathrm{S}} \mathrm{O}_{4}{ }^{3}-\mathrm{A}_{\mathrm{S}} \mathrm{O}_{2}$-por hidrógeno sulfuro transcurre muy lentamente, la precipitación se lleva a cabo en frío, una parte de los iones se transforman a $\mathrm{A}_{\mathrm{S}} \mathrm{O}_{2}$ -

$$
\mathrm{As}_{\mathrm{S}} \mathrm{O}_{4}{ }^{3+}+\mathrm{H}_{2} \mathrm{~S}+2 \mathrm{H}^{+} \rightarrow \mathrm{As}_{\mathrm{s}} \mathrm{O}_{2}^{-+} \mathrm{S}+\mathrm{H}_{2} \mathrm{O}
$$

que da lugar a la formación de una mezcla de $\mathrm{A}_{\mathrm{S} 2} \mathrm{~S}_{5}$ y $\mathrm{A}_{\mathrm{S} 2} \mathrm{~S}_{3}$.

Los metales alcalino térreos están combinados con arseniatos $(V)$, ccmo sales escasamente solubles, en consecuencia el arsénico tiene tendencia a formar frecuentemente precipitado en asociación con los carbonatos y redes coloidales de $\mathrm{Fe}$ (III).

En la tabla No. 5 se aprecia el arsénico soluble al tener el agua en contacto con la caliza y modificar su $\mathrm{pH}$ al formar parte de las redes de los complejos polinucleares de Fe (III). La determinación se realizó porel método de la plata dietil carbamato.

TABLA No. 5: Arsénico soluble en agua del río Tarucachi tratada con caliza

\begin{tabular}{|c|c|c|}
\hline $\mathrm{pH}$ & Tiempo $(\mathrm{min})$ & As soluble $\mathrm{mgdm}^{-3}$ \\
\hline 3,35 & 0 & 0,050 \\
5,90 & 0,5 & 0,028 \\
6,50 & 1,0 & 0,018 \\
6,65 & 1,5 & 0,009 \\
6,80 & 3,0 & 0,005 \\
6,85 & 5,0 & 0,005 \\
6,90 & 10,0 & 0,005 \\
7,00 & 15,0 & 0,005 \\
\hline
\end{tabular}

Fuente : Análisis LFQ - UNJBG.

\section{ANALISIS DE LOS RESULTADOS}

a) Utilizando $\mathrm{CaCO} 3$, (caliza), en el laboratorio como en el canal experimental construido en la quebrada de Kejane - Tarucachi se demuestra que en períodos diferentes se logra la neutralización del pH. En el laboratorio en el plazo de 15 minutos aproximadamente se llega a un $\mathrm{pH}$ neutro.

b) El hierro comienza su transformación a pH 3,35-4,0 aFe(III).

c) El equilibrioácido-base para el hierro (III) está dado por las ecuaciones:

$$
\begin{aligned}
& {\left[\mathrm{Fe}\left(\mathrm{H}_{2} \mathrm{O}\right)_{5}\right]^{3++} \mathrm{H}_{2} \mathrm{O} \rightarrow\left[\mathrm{Fe}\left(\mathrm{H}_{2} \mathrm{O}\right)_{5}(\mathrm{OH})\right]^{2+}+\mathrm{H}_{3} \mathrm{O}^{+}} \\
& {\left[\mathrm{Fe}\left(\mathrm{H}_{2} \mathrm{O}\right)_{5}(\mathrm{OH})\right]^{2+}+\mathrm{H}_{2} \mathrm{O} \rightarrow\left[\mathrm{Fe}\left(\mathrm{H}_{2} \mathrm{O}\right)_{4}\right]^{+}+\mathrm{H}_{3} \mathrm{O}^{+}}
\end{aligned}
$$


Pueden continuar transferencias similares. De acuerdo al pHencontrado en el agua y confrontada con la bibliografía al formarse sales férricas se produce la disminución del $\mathrm{pH}$.

d) Los complejos de hierro (III) hidroxo tienen una enorme tendencia a polimerizarse, produciendo reacciones como (1), (2) y (3).

$$
2\left[\mathrm{Fe}\left(\mathrm{H}_{2} \mathrm{O}\right)_{5}(\mathrm{OH})\right]^{2+} \rightarrow\left[\mathrm{Fe}_{2}\left(\mathrm{H}_{2} \mathrm{O}\right)_{6}(\mathrm{OH})_{2}\right]^{4+}+2 \mathrm{H}_{2} \mathrm{O}
$$

Los dos iones metálicos se encuentran probablemente ligados mediante puentes hidroxo.

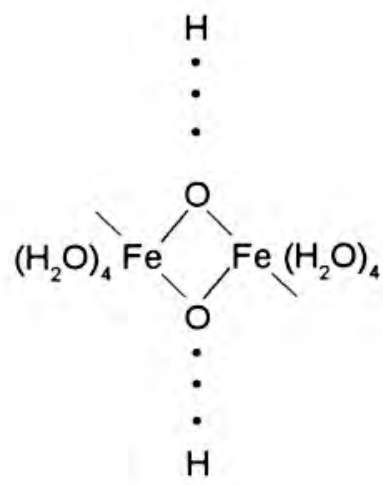

Esta unidad polimérica sufre reacciones hidrolíticas adicionales formando complejos mayores de hidróxidos, los cuales originan otros puentes hidroxo que dan lugar al polimero coloidal.

e) La sílice en suspensión y otras sales insolubles hace que se rompan las redes de los polímeros

\section{BIBLIOGRAFIA}

Fair-Okun, 1979.Purificación de Aguas. Tomos II, Ed. Limusa.

Gastany, G. 1975. Prospección y explotación de las aguas subterráneas. Ed. Omega.

Molina, L., 1989. Interacción y eliminación de arsénico y hierro de agua del río Tarucachi. Tesis hidroxo coloidales y formen precipitados insolubles de hierro (III) hidroxo hidratados.

f) Las especies hidrolizadas y no el hierro (III) libre causan la inversión de la carga.

g) La modificación delpH establece diferentes equilibrios arsénicos como $\mathrm{A}_{\mathrm{s}} \mathrm{O}^{3}$ que, al reaccionar con los elementos alcalino térreos, $y$ al incrementarse las redes coloidales del Fe (III), son capturados y se precipitan.

h) En el canal experimental debe construirse filtros cada cierta distancia para eliminar los glomérulosy permitir que la caliza en las siguientes etapas, tenga una mayor eficiencia en la modificación del $\mathrm{pH}$ y las reacciones y equilibrios anteriormente mencionados.

i) La limpieza del canal experimental se hace manualmente, en periodos que varían de acuerdo a los periodos de estiaje y lluvia

\section{CONCLUSION}

El trabajo muestra que la eliminación del arsénico de las aguas naturales del río Tarucachi es gobemada por el hierro (III) de los complejos polinucleares formados al modificar el pH con $\mathrm{CaCO}_{3}$ (caliza) y no por el ión $\mathrm{Fe}^{3+}$ libre, ya que estos complejos absorben fuertemente en su red estructural una porción variable de diferentes iones quedentro de los límites de neutralidad eléctrica precipitan, y, porfiltración, son eliminados purificando así las aguas de origen geotermal con elevadas concentraciones de hierro y arsénico.

\section{MSC - UNSA}

Molina, L., Alvarez, R. y otros, 1992. Canal experimental para la eliminación de $\mathrm{Fe}$ y AS de las aguas del rio Tarucachi. Informe UNJBG.

World Health organization, 1971. International Standars For Drinkins Water. Tercera edición. 